\title{
Los senderos productivos de la bioeconomía: El caso de Honduras
}

\section{The productive paths of the bioeconomy: The case of Honduras}

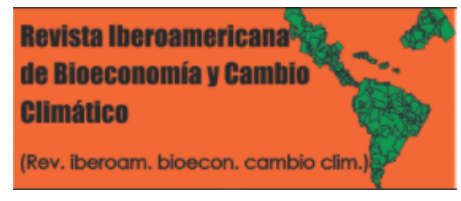

\author{
Colón-García, A. P; Catari-Yujra, G.; Alvarado, E.3, E.; Editor \\ Academico Dr. Roberto A. Berrios Zepeda
}

\author{
A. P Colón-García \\ adelfa.colon@unah.edu.hn \\ Universidad Nacional Autónoma de Honduras, \\ Honduras \\ G. Catari-Yujra \\ gusman.catari@unah.edu.hn \\ Universidad Nacional Autónoma de Honduras, \\ Honduras
}

(iD) E. Alvarado, E.3

enrique.alvarado@catie.ac.cr

Centro Agronómico Tropical de Investigación y

Enseñanza (CATIE), Gestión del conocimiento.,

Costa Rica

Editor Academico Dr. Roberto A. Berrios Zepeda

roberto.berrios@ce.unanleon.edu.ni

Universidad Nacional Autónoma de Nicaragua, León.,

Nicaragua

Revista Iberoamericana de Bioeconomía y Cambio

Climático

Universidad Nacional Autónoma de Nicaragua, León, Nicaragua ISSN-e: 2410-7980

Periodicidad: Semestral

vol. 7, núm. 14, 2021

czuniga@ct.unanleon.edu.ni

Recepción: 16 Abril 2021

Aprobación: 27 Noviembre 2021

URL: http://portal.amelica.org/ameli,

jatsRepo/394/3941761011/index.html

DOI: https://doi.org/10.5377/ribcc.v7i14.12820

Autor de correspondencia: adelfa.colon@unah.edu.hn
Resumen: Los medios de sustento de la sociedad en general y los ecosistemas naturales están siendo afectados negativamente por el cambio climático. Ante esto, surgen alternativas como la bioeconomía que busca disminuir el impacto ambiental y el cambio climático a través de sus principales senderos. De esta forma, el propósito de este estudio fue indagar el potencial de desarrollo de la bioeconomía en Honduras, considerando el aporte del sector agrícola al Producto Interno Bruto (PIB) del país. El estudio se desarrolló durante el año 2021 y el enfoque utilizado fue la investigación bibliográfica, a través de la revisión sistemática de literatura. Los resultados muestran que durante el período del 2015 al 2020, la contribución del sector agrícola en Honduras se mantuvo en 14\%, ocupando el tercer lugar después del sector de industria manufacturera y la intermediación financiera. Ahora bien, en relación a la bioeconomía fue posible establecer contribuciones puntuales en los siguientes senderos: a) Valoración de la diversidad biológica, b) Eco intensificación, c) Aplicaciones de biotecnología, d) Servicios eco sistémicos, e) Competitividad de las cadenas de valor, y f) Biorefinería y bioproductos. Como conclusión se tiene que el tema de bioeconomía en Honduras se inclina hacia la biotecnología y la biorefinería. Aunque ambos senderos involucran tecnologías poco accesibles a pequeños productores agropecuarios. Por lo tanto, es primordial el desarrollo participativo de políticas y estrategias de bioeconomía para el área de productos y servicios, lo que mejoraría la competitividad y desarrollo sostenible del país.

Palabras clave: Cambio climático, Bioproductos, Biotecnología, Eco intensificación, Biodiversidad.

Abstract: Climate change has a negative effect on ecosystems, livelihoods, and society in general. Because of this, alternatives arise like the bioeconomy that seeks to reduce the environmental impact and climate change through its main pathways. In this regard, the objective of this research was to evaluate the contribution of the agricultural sector to the Gross Domestic Product (GDP) of Honduras and the development potential of the bioeconomy in the country. The research was carried out in Honduras, during 2021 and the approach used was qualitative, through a systematic review of the literature. The main results show that during the period between 2015 and 2020 the contribution of the agricultural sector 
remained at $14 \%$, ranking third after the manufacturing industry and financial intermediation sector. However, regarding the bioeconomy, it was possible to establish specific contributions in the following pathways: a) Valuation of biodiversity resources, b) Eco intensification, c) Biotechnology applications, d) Ecosystem services, e) Efficiency of value chains, and f) Bio refineries and bioproducts. As a conclusion, the issue of bioeconomy in Honduras leans towards biotechnology and biorefineries. Although both paths involve technologies that are not very accessible to small agricultural farmers. Therefore, the participatory design of bioeconomy policies and strategies for the products and services sector is essential, which would improve the competitiveness and sustainable development of the country.

Keywords: Climate change, Bioproducts, Biotechnology, Eco intensification, Biodiversity.

\section{INTRODUCCIÓN}

De acuerdo con Ruiz Muller et al. (2020), Sánchez et al. (2018) en años recientes, el tema de cambio climático ha sido introducido en las agendas nacionales de diferentes países de forma progresiva; sin embargo, es necesario seguir trabajando en la socialización y construcción de capacidades regionales y locales sobre el tema; ya que los productores y otros actores clave si exponen que perciben los cambios en los patrones climáticos Blanco-Orozco et al. (2014), pero usualmente no los asocian al fenómeno global; aun cuando los efectos negativos del cambio climático repercuten directamente en los ecosistemas, medios de vida de la población, y la actividad macroeconómica en general López-González et al. (2015). En este sentido, depender de combustibles fósiles, como el carbón y el petróleo, y utilizar materias primas de manera ineficiente sitúan a la población en escenarios que amenazan su sostenibilidad en el mediano y largo plazo. Sumado a lo expuesto, el crecimiento de la población humana mundial intensificará todavía más la competencia por los recursos naturales y añadirá presión al medio ambiente (Domínguez et al., 2019). Por lo anterior, es importante profundizar en modelos de crecimiento que faciliten el uso eficiente de recursos biológicos MarinerosOrantes et al. (2015).

Ante este panorama cobra fuerza la denominada bioeconomía, la cual, según la definición de Rodríguez et al. (2017),Zuniga-Gonzalez et al. (2014) se basa en el proceso de producción e incremento del consumo de bienes y servicios que se derivan de la transformación y utilización directa de recursos biológicos de manera sostenible, incluyendo los residuos generados en las etapas de producción, trasformación, y consumo; tomando en consideración la capacidad de comprender procesos y principios de índole biológico y el uso de tecnologías apropiadas y de fácil implementación acorde con la información y transformación de los recursos; la cual, como forma de producción, busca disminuir el impacto ambiental principalmente en áreas rurales y proteger la base de los recursos naturales Zuniga-Gonzalez, (2020).

Por lo tanto, el presente artículo analiza el contexto actual de la bioeconomía en Honduras en función de sus diversos senderos.

NotAS DE AUTOR

adelfa.colon@unah.edu.hn 


\section{Metodología}

Para la investigación se realizó una búsqueda de documentación sobre Bioeconomía en Honduras incluyó artículos científicos, publicaciones del gobierno, y otras fuentes que permitieron conocer el estado del arte del tema en el país. La búsqueda de esta información fue principalmente a través de buscadores de Internet complementado con consulta a expertos (Rangel-Cura et al., 2015; Zuniga-Gonzalez et al., 2014a;Gonzalez, 2011).

\section{REVISIÓN DE LITERATURA}

Según la FAO (2018), la naturaleza transversal de la bioeconomía ofrece una opción primordial para tratar los siguientes retos sociales que se encuentran interconectados de forma integral: a) El uso exhaustivo de combustibles fósiles, b) La falta de acceso a recursos naturales, c) La inseguridad alimentaria, y d) El cambio en los patrones del clima; además, es capaz de contribuir a un desarrollo económico sostenible. En relación a esto y tal como se muestra en el anexo 1 , se han identificado seis "senderos productivos" de la bioeconomía (Trigo et al., 2013).

\section{Contexto de país}

Honduras es un país que posee una economía abierta, pequeña y de ingreso bajo-medio, tiene una superficie de $112.492 \mathrm{~km} 2$ y su población en el 2020 era de 9,3 millones de habitantes (INE, 2020). Durante el mismo año, su Producto Interno Bruto (PIB) per cápita fue de L. 62,952.00 equivalente a US\$ 2,563.00 (BCH, 2020). La canasta exportadora presenta poca diversificación y se concentra principalmente en bienes primarios de escaso valor agregado, lo cual incrementa su fragilidad ante la volatilidad de precios internacionales y a eventos climáticos adversos, que lo convierten en un país muy vulnerable.

Los cambios asociados al cambio climático que modifican la temperatura y precipitación promedio, y eventos periódicos como el fenómeno de El Niño, han generado impactos negativos en la producción agropecuaria, por tanto, en la seguridad alimentaria; así como un aumento de las enfermedades, y la presencia cada vez más frecuente de incendios forestales (Medina, 2018).

Los cambios de uso de suelo, entre estos la deforestación, representaron el 31\% de las emisiones de gases de efecto invernadero para el año 2015 (Mi Ambiente, 2015). Concretamente, el país presenta un alto nivel de deforestación, y sus consecuencias en áreas de bosque latifoliado son significativas; además, en áreas de bosque de pino el ataque del gorgojo devastó más de 500.000 hectáreas entre 2013 y 2016 (Marques Almeida et al 2019). Por otra parte, y de acuerdo al BID (2019), se estima que el $47 \%$ de la población del país vive en zonas altamente susceptibles a riesgos naturales Figura 1 y 2 ..

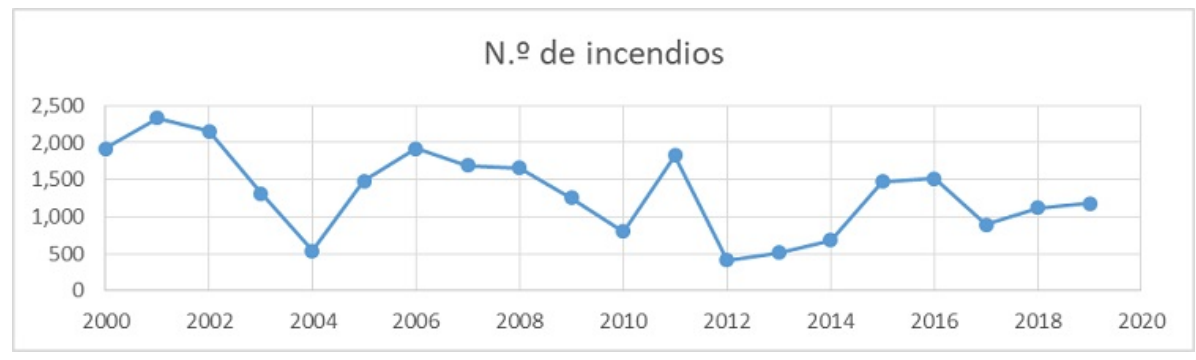

FIGURA 1.

Número de incendios reportados en Honduras entre el 2000-2020.

Fuente: BCH (2020) 


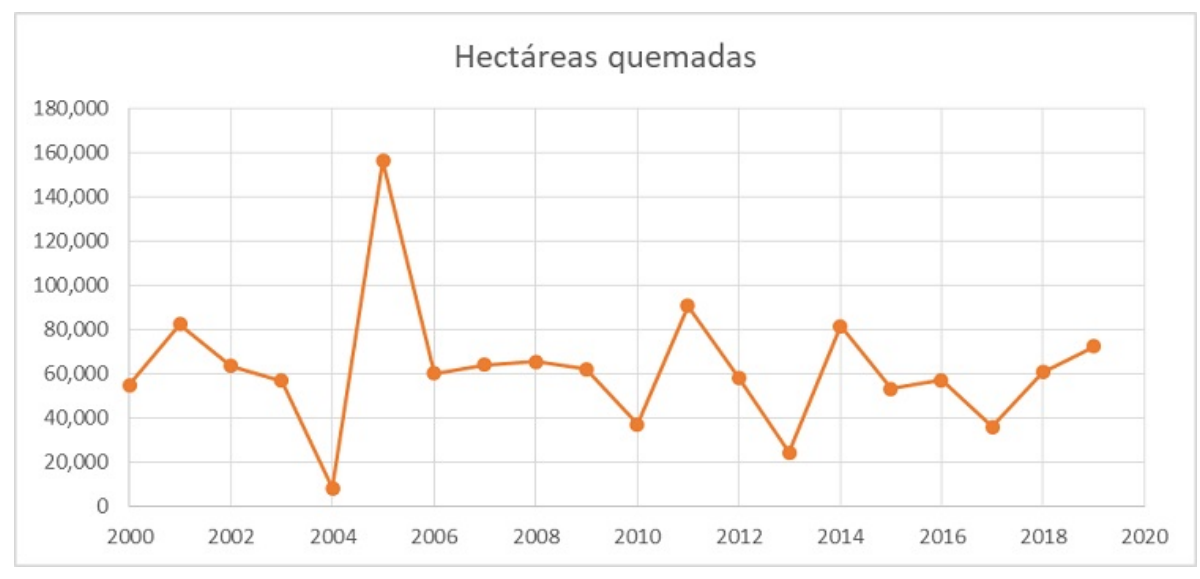

FIGURA 2.

Número de hectáreas quemadas.

Fuente: $\mathrm{BCH}(2020)$.

En la última década, del total de incendios reportados el $64 \%$ han sido provocados por 'mano criminal', $11 \%$ corresponden a actividades agropecuarias y el $25 \%$ restante se distribuyen entre cacería, pesca, extracción de miel, caminantes nocturnos, entre otros (Medina Moncada, 2018).

\section{Contribución del sector agricola al PIB de Honduras}

Durante el período del 2015-2020, la contribución del sector agrícola se mantuvo en 14\%, ocupando el tercer lugar después del sector de industria manufacturera y la intermediación financiera Figura 3.

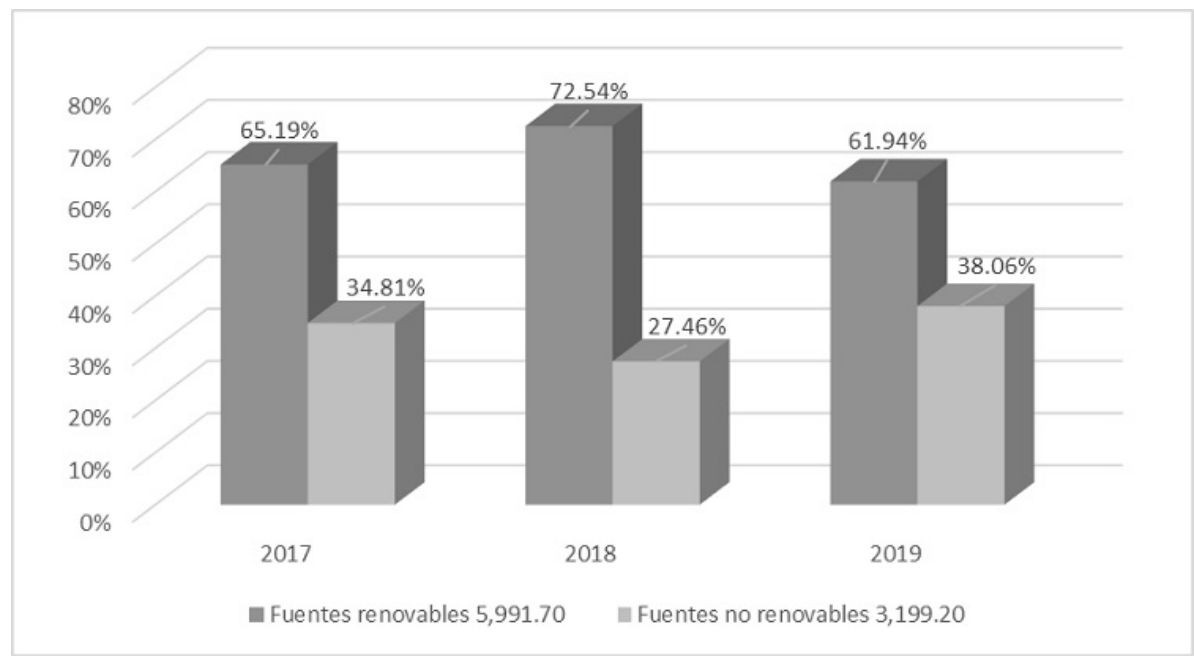

FIGURA 3.

Producto Interno Bruto (PIB) por actividad económica para el período 2015-2020*.

Fuente: Elaboración propia con información del BCH. ${ }^{*}$ Nota: Valores Constantes (Millones de Lempiras).

El sector primario es el que más genera productos, empleo, ingresos, y exportaciones a la economía del país; igualmente, es el que provee una diversidad de servicios ecosistémicos o ambientales. Sin embargo, muchos de los procesos generan gran cantidad de biomasa que no está siendo aprovechada. Por lo tanto, para poder establecer cómo este sector puede contribuir con la bioeconomía en Honduras, es necesario estimar la generación de residuos orgánicos en cada proceso productivo. Para ello, es necesario realizar un control 
documentado de la biomasa residual generada en las unidades productivas, y establecer los sistemas más adecuados de aprovechamiento energético de residuos.

\section{Hondurasy los senderos productivos de la bioeconomía Valoración de los recursos de base biológica}

Honduras presenta un alto nivel de biodiversidad que siempre ha sido aprovechada por el ser humano. De esta forma, el potencial que podría tener este sector en la bioeconomía se basa en el uso de la biomasa que proviene de recursos biológicos (microorganismos, plantas y animales), sus genes y la variedad de ecosistemas que son parte de la naturaleza.

De acuerdo con WICE (2002), citado por Mi Ambiente (2017), Honduras se encuentra caracterizado como un país mayormente montañoso y con distintos niveles de clima, lo que promueve una amplia variedad de zonas de vida y alta diversidad biológica. Para tal caso, en el país es posible identificar alrededor de 214 plantas endémicas; además, se encuentran aproximadamente 770 especies de aves, de las cuales cinco se encuentran presentes en la lista mundial de animales en peligro de extinción, y alrededor de 59 habitan en ecosistemas con un alto nivel de deterioro. Sumado a lo anteriormente expuesto, en Honduras se encuentra una de las más importantes y extensas reservas mundiales de material genético de pino tropical, que son clave para la silvicultura y que, en la actualidad, toman mayor importancia al ser sumideros naturales de carbono.

Ahora bien, de acuerdo a Ordoñez y Ferrufino (2020), en Honduras existen diferentes esquemas de aprovechamiento de la biodiversidad, se debe a la variedad cultural que predomina en todo el territorio; sin embargo, se ha dado poca importancia al rescate, intercambio y potenciación de los conocimientos tradicionales; por lo tanto, es primordial sistematizar el conocimiento ancestral, étnico y/o tradicional para promover la conservación de la biodiversidad. De lo contrario, como lo expresa Medina (2018), la pérdida de biodiversidad en Honduras será recurrente por factores determinantes como el inadecuado uso del suelo, la infraestructura de carreteras, la fragmentación de áreas naturales y el cambio climático.

Además, aunque Honduras es parte del Convenio sobre la Diversidad Biológica, no cuenta con un marco legal actualizado, efectivo y eficiente que regule el uso de recursos genéticos, la correcta repartición de beneficios, la gestión del conocimiento y transferencia tecnológica, y que defina los lineamientos para el desarrollo de investigaciones. En concreto, el mayor provecho que puede obtenerse de este sendero productivo podría estar representado por el sector turismo, ya sea por la valoración económica del paisaje como insumo para el ecoturismo y el establecimiento de esquemas de Pago por Servicios Ambientales (PSA) Alvarado (2006) Igualmente, existe la oportunidad de conservar hábitats de especies endémicas y en peligro de extinción como el colibrí esmeralda de Honduras (Amazilia luciae) a través de esquemas que involucren los servicios ecosistémicos de la biodiversidad, situación que también debe promoverse en la región de la Mosquitia del país.

Eco intensificación

Este sector productivo, según los proponentes de la bioeconomía, se vincula al uso de microorganismos que permitan incrementar los índices productivos; sobre todo, a través de la implementación de bio-inoculantes, el establecimiento de sistemas agroforestales y silvopastoriles, la biorremediación y el uso de buenas prácticas (Zúniga-Gonzalez et al., 2014).

En este sentido, entre las prácticas agroecológicas más representativas de Honduras se encuentra el modelo de asocio con la guama (Inga. spp.) en la región del bosque húmedo del país; sobre todo, en el Departamento de Atlántida (Hands, 2021). Este cultivo permite la recuperación de terrenos al fijar grandes cantidades de nitrógeno $\left(\mathrm{N}^{2}\right)$, generar hojarasca que evita la proliferación de malezas y evitar la erosión; además, sirve de sombra intermedia en sistemas agroforestales con cacao (Theobroma cacao) y/o café (Coffea arabica), y es clave para mejorar la seguridad alimentaria de la población, ya que su fruto es comestible. 
Otro sistema representativo y originario de Honduras es el Quesungual, que básicamente se caracteriza por el uso de la labranza cero en pequeñas parcelas en laderas con pendientes muy pronunciadas, y con problemas de sequía y erosión. Generalmente, este sistema se implementa en zonas áridas del país, con cultivos como maíz (Zea mays), frijoles (Phaseolus vulgaris) y sorgo (Sorghum sp.) y otras hortalizas, pero incluyendo un componente forestal, de árboles frutales y/o maderables, que permite el abastecimiento de frutas y madera para leña o muebles. Esto genera un equilibrio en el sistema productivo, lo que permite incrementar los rendimientos, contribuir a la seguridad alimentaria, aumentar la resiliencia de los sistemas socio-ecológicos y fijar carbono como medida de mitigación del cambio climático. De acuerdo con Cherret y Welches (2019), el nombre de este sistema se deriva de una región del departamento de Lempira. Además, autores como Acuña et al. (2020), establecen que al utilizar el sistema Quesungual se obtiene mayor biomasa en la superficie de los suelos y se produce mayor movimiento biológico.

Por otra parte, en el país se identifican otras prácticas como el uso de barreras vivas y muertas, sistemas de cosecha de agua con micro riego, uso de terrazas, labranza de conservación, acequias, entre otras (PESA, 2005). Finalmente, como lo indica Borrego Camacho (2020), es clave tener presente el conocimiento ancestral de las etnias para mejorar la producción y hacer frente a los problemas climáticos.

En este sentido, este sendero productivo podría representar una oportunidad para los pequeños productores agropecuarios del país; no obstante, es necesario que se realicen análisis costo- beneficio para cada práctica o sistema, con el fin de exponer a las agencias de cooperación, implementadores, productores y banca en general, los beneficios técnicos y económicos del uso de cada práctica, lo que también permitiría establecer mejores sistemas de evaluación, integrar estas prácticas en los mecanismos financieros de la banca para reducir el riesgo climático y gestionar proyectos bancables.

Aplicaciones de biotecnología

La aplicación de la ingeniería genética en la agricultura es un tema polémico y se recomienda realizar evaluaciones periódicas para conocer su efecto o no en los sistemas socio-ecológicos. No obstante, los promotores de estas tecnologías indican que la biotecnología (Zuniga-Gonzalez et al., 2014) permite desarrollar variedades mejoradas de plantas. Básicamente, facilita que existan variedades tolerantes a plagas, enfermedades y condiciones climáticas adversas como la sequía, entre otros aspectos. Adicionalmente, autores como Chavarria et al. (2021), exponen que contribuyen a la generación de impactos positivos en la sostenibilidad ambiental; sin embargo, deben tenerse en cuenta los potenciales efectos negativos para los humanos, así como los riesgos ecológicos vinculados a la creación de resistencia, por ejemplo la aparición de 'super malezas', y alteración de la red trófica (Chen Zhang, et al. 2016).

Cabe mencionar que las plantas que provienen de un proceso biotecnológico en que se involucra la transferencia de genes se denominan transgénicas u Organismos Modificados Genéticamente (OMG) (Agro-Bio, 2016). En Honduras, durante la mitad de la década de los 90s se establecieron lineamientos para promover el progreso e incorporación de la biotecnología, pero como mencionan Catari y Martínez (2020), las políticas públicas vinculadas a este tema se encuentran en la misma línea que las establecidas por organismos internacionales, a pesar de que en el proceso de diseño, validación y socialización de estos instrumentos regulatorios existió la opción de integrar lineamientos de especialistas del país para que las políticas se adecuaran a la realidad nacional. Es así, que en 1998 fue aprobado el Reglamento de Bioseguridad del país, con un mayor enfoque para maíz (Zea mays) transgénico, el cual fue emitido por la Secretaria de Agricultura y Ganadería (SAG) con el propósito de establecer el proceso de regulación de los transgénicos. En relación a lo expuesto, Honduras produce y comercializa maíz transgénico, lo que ha permitido que del año 2002 al 2016 los ingresos por concepto agrícola hayan sido de US\$ 11.5 millones (Ridher et al. 2020).

No obstante, la adopción de esta tecnología es baja, a pesar de las campañas masivas de promoción de la misma. En este contexto, se considera que los sistemas basados en principios agroecológicos tienen mayores ventajas en comparación a los basados en biotecnología (transgénicos) por dos razones: son sostenibles en 
el tiempo y porque consideran el agroecosistema de una manera holística, lo opuesto a una concepción reduccionista. Por tanto, este sendero tiene potencial, pero si se brinda prioridad a los sistemas agroecológicos.

Servicios eco sistémicos

A nivel general, estos servicios se originan de las funciones conjuntas de los ecosistemas; por lo tanto, es necesario que exista una clara delimitación de los mismos, que se establezcan los servicios que proveen (hídricos, belleza escénica, conservación de la biodiversidad, secuestro y fijación de carbono, entre otros), que se determinen los beneficiarios directos y la forma sostenible de conservarlos; sobre todo, a nivel de buenas prácticas agrícolas. De lo contrario, la agricultura convencional que involucra el uso intensivo de agroquímicos y el establecimiento de monocultivos, entre otros aspectos, afectará o eliminará la provisión de estos servicios para los seres humanos. Para tal caso, como indica Gómez et al. (2018), acciones como la labranza mecanizada tienen un impacto directo en la estructura y poros del suelo, afectando así el servicio ambiental hídrico que proviene de la parte alta de las microcuencas o zonas productoras de agua, ya que este tipo de prácticas agrícolas no sostenibles afectan el proceso de infiltración y almacenamiento de este líquido, ya que se genera escorrentía, erosión del suelo y disminución de las cantidades de agua para uso doméstico o agrícola. Lo anterior, indica que existe una creciente necesidad de rediseñar los esquemas productivos y el tipo de prácticas implementadas, con el fin de liberar presión o preservar los servicios eco sistémicos (Bolaños, 2020).

Es así, que la literatura sobre el tema ha demostrado que la valoración económica de la oferta y demanda de los servicios ecosistémicos contribuye a establecer un sistema de mercado que permita pagar para la conservación de dichos servicios e implementar prácticas sostenibles de acuerdo a cada servicio ambiental. Sin embargo, existen factores como el cambio climático que afectarán estas funciones de los ecosistemas, a menos que no se considere la valoración económica y el establecimiento de esquemas de Pago por Servicios Ambientales (PSA) a escala global entre los países industrializados y los que poseen mayor riqueza natural.

De acuerdo con Silva (2019), que cita a Alvarado (2008), el país no cuenta con lineamientos ni estrategias efectivas y eficientes para reconocer el valor de los servicios ecosistémicos, dado que se consideran una falla de mercado porque no existe un valor o precio para su conservación. Aun así, en el país se han articulado algunos esquemas de PSA; básicamente, existen 26 esquemas para la conservación del servicio ambiental hídrico de uso doméstico, 10 esquemas para la conservación del servicio ambiental hídrico para generar energía, dos esquemas vinculados al servicio ambiental de secuestro y fijación de carbono y una para articular el ecoturismo y conservar el paisaje. No obstante, estos datos deben actualizarse y socializarse con los tomadores de decisión, para crear conciencia sobre la importancia de desarrollar mercados que contribuyan a conservar los ecosistemas y sus servicios.

\section{Eficiencia de las cadenas de valor}

A nivel general, las cadenas de valor agrícola son poco eficientes, ya que generan residuos orgánicos de cosechas y una amplia gama de subproductos originados del procesamiento y la comercialización de los alimentos; similar situación sucede en el sector forestal. Por lo que se produce una gran cantidad de biomasa que en general no es aprovechada por la economía convencional o lineal, y debido a esto es preponderante el cambio hacia una economía circular donde se cierren los ciclos productivos aprovechando todos los residuos provenientes de las actividades productivas. En adición a lo anterior, la población de asentamientos rurales y urbanos genera biomasa en altas cantidades, incluyendo los residuos cuyo destino final son los vertederos y/o basureros municipales.

$\mathrm{Al}$ igual que en muchos países de Latinoamérica, en Honduras existe poco interés para el aprovechamiento de la biomasa que proviene del sector industrial y productivo. Solamente, existen iniciativas privadas para cerrar los ciclos de producción, pero a nivel de políticas, estrategias y planes locales no existen lineamientos 
claros que permitan aprovechar estos residuos o biomasa para mejorar la eficiencia de las cadenas de valor. (Macías Aragonés et al., 2020).

De acuerdo a la FAO (2018), anualmente en el mundo son generadas 3.3 gigatoneladas de Gases de Efecto Invernadero (GEI) debido a los residuos de los procesos productivos agroalimentarios, debido a esto, este sector es el tercero en cantidad de emisiones. De esta forma, al evaluar económica y ambientalmente estos residuos y su adecuada gestión, permitiría mejorar los márgenes de utilidad de las cadenas de valor y contribuir con la sostenibilidad ambiental del planeta.

En relación a lo expuesto, la Dirección de Ciencia y Tecnología Agropecuaria (DICTA), y su dependencia PRONAGRO, han organizado diferentes cadenas productivas en al país para facilitar el desarrollo de cadenas de valor, entre las cuales destacan la cadena de pesca y acuicultura, cadena avícola (pollos de engorde y ponedoras, crías cinegéticas como gansos, pavos, y avestruces en menor escala), cadena bovina (leche y carne), cadena de café y cacao, cadena de granos básicos, cadena hortifrutícola, cadena apícola y la cadena de cultivos bio-energéticos (palma africana y la caña de azúcar) (Moreno Banegas, 2012).

Para el caso en la cadena del café existen experiencias de generación de bioproductos como el licor de café, cerveza artesanal y los biosensoriados, además de ofrecer bioservicios de agroturismo (Fortín, 2021). De esta forma, se considera que este es otro de los senderos productivos a los cuales Honduras debe apostar, ya que existe gran cantidad de residuos en las cadenas antes mencionadas; sobre todo, en los sectores agroforestales de café y cacao, y el de palma africana y caña de azúcar; por lo que es recomendable promover estudios de factibilidad para la gestión de la biomasa e implementar esquemas de economía circular que faciliten la eficiencia de las cadenas de valor.

\section{Biorefinerias y bioproductos}

Una biorrefinería hace referencia a un lugar con las condiciones de infraestructura y equipo apropiado para transformar biomasa en otros productos como: biogás, bioetanol, biofertilizantes, combustibles y/o energía, entre otros. De acuerdo a Aguilar Rivera (2014), las biorrefinerías son semejantes a las refinerías petroleras, ya que su objetivo es el de transformar productos primarios en otros más elaborados y de uso directo para la población.

En 1998, en Honduras se elaboró la Ley de Incentivos a la Energía Renovable, lo cual convirtió a este país en el segundo en Centroamérica en contar con este tipo de marco legal; adicionalmente, en el 2007 el Congreso Nacional aprobó la Ley de Incentivos a la Generación Eléctrica con Energía Renovable (Decreto 70-2007). Dicha ley permitió la gestión de proyectos de energía renovable en todo el país; sobre todo, los vinculados a la cogeneración de energía eólica e hidroeléctrica. Sumado a lo expuesto, en el 2013, a través del Decreto 138-2013 se reformó el Decreto 70-2007 para incluir e impulsar la energía solar fotovoltaica.

Debido al impulso del marco legal en materia de energía renovable, en el período 2017 al 2019, la matriz energética del país se encontraba conformada en un $60 \%$ por energía renovable, siendo su punto más alto durante el 2018 cuando alcanzó un 75\% de contribución a la matriz energética nacional Figura 4 


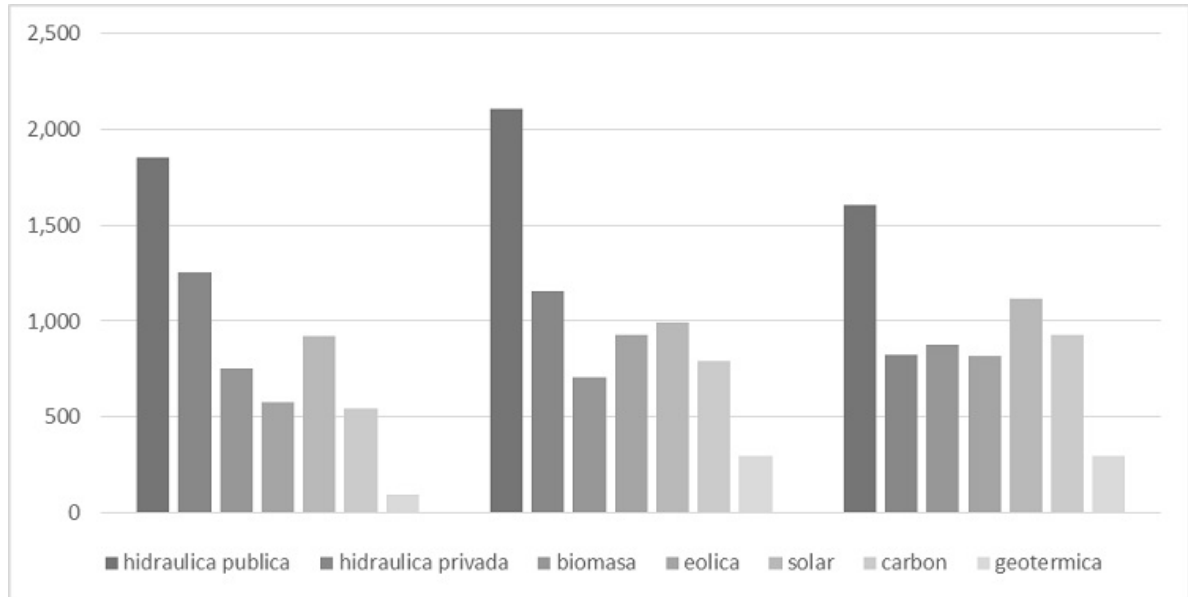

FIGURA 4.

Generación de energía por tipo de fuente (Unidades MW)

Fuente: BCH (2020)

De esta energía renovable la mayor participación correspondió a la energía hidráulica de carácter público con la tercera parte de la contribución total en 2017; sin embargo, esta contribución bajó a menos de la cuarta parte en 2019. La que mostró un mayor aumento fue la energía solar y la que se basa en carbón. La participación de la energía derivada de la biomasa se mantiene en un rango entre 10 a 14\% Figura 5.

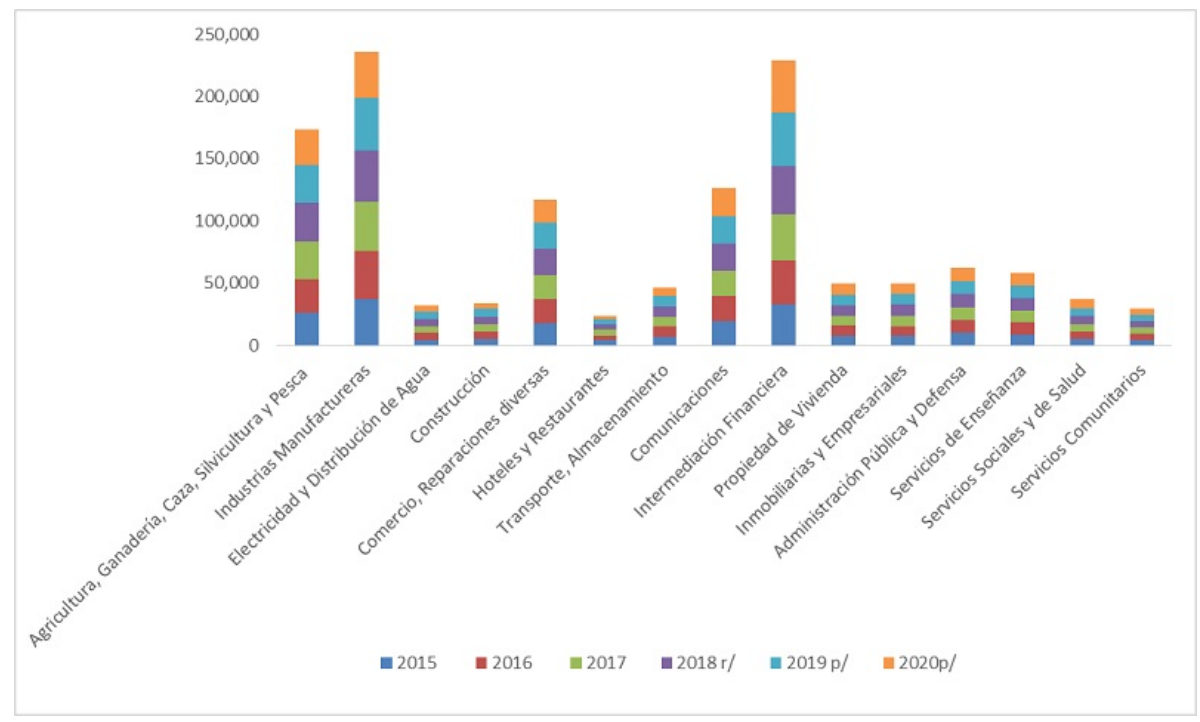

FIGURA 5

Tipo de energía renovable producida 2017-2019 (Unidades KW)

Fuente: $\mathrm{BCH}(2020)$

\section{Conclusiones}

Cuando se busca información de la bioeconomía en Honduras, la inclinación es muy marcada hacia dos de los senderos productivos: la biotecnología, especialmente en los organismos genéticamente modificados y las biorrefinerías. Ambos senderos involucran principalmente tecnología poco accesible a pequeños productores agropecuarios. Sin embargo, en el país existen organizaciones de productores que están incursionando en otros sectores de la bioeconomía como ser eco intensificación (Sierra-Figueredo et al., 2015) a partir de prácticas como manejo sostenible del suelo, y sistemas agroforestales y silvopastoriles. 
La implementación de la bioeconomía afronta muchos desafíos (Milán \& Zúniga-Gonzalez, 2021), y su enfoque debe ser dirigido a mejorar los índices de seguridad alimentaria, a gestionar de mejor forma los cambios en los patrones climáticos en el mediano y largo plazo, y manejar los recursos sosteniblemente, promoviendo los diferentes usos de las materias primas de biomasa para beneficio de la sociedad (BlancoOrozco et al., 2016).

El diseño de políticas de bioeconomía en Honduras debe ser liderada por el sector público (MarineroOrantes et al., 2015), por poseer representatividad política. Actualmente, el impulso de la bioeconomía es dada por entes externos regionales como el (IICA, 2020) que a su vez promueve políticas diseñadas y/o adoptadas por países industrializados. El aporte de dichos entes es importante sin duda, sin embargo, el ente coordinador debe ser el Estado. Como se ha descrito en el documento, en el país existe potencial para algunos de los denominados senderos de la bioeconomía, como la eco-intensificación (por ejemplo sistemas agroecológicos) pero el diseño de políticas y estrategias para su despliegue debería ser endógeno, encabezada por el Estado, garantizando la participación multisectorial e inclusión de los sectores que menos han sido favorecidos por las políticas económicas del libre mercado implementado en las últimas décadas, lo cual ha conducido que Honduras sea uno de los países más desiguales en cuanto a ingresos per cápita en la región y con niveles de extrema pobreza por encima del $40 \%$.

Actualmente no hay suficiente debate sobre lo que es la bioeconomía (Rodrigueez, 2020, 2019), sobre sus beneficios y sus riesgos, y cómo se va a medir (desarrollo de indicadores). El debate debería ser transversal a todas las disciplinas del conocimiento, desde ciencias sociales hasta ciencias naturales (Vargas-Hernández, 2018). En el debate se debe considerar conceptos como la 'economía verde', 'economía circular', 'soberanía alimentaria' entre otros, también consideraciones sobre una relación más justa entre los países con economías desarrolladas y otros con emergentes o en desarrollo, e intentar responder a preguntas como ¿No será que los países con economías desarrolladas están 'forzando' a países con economías emergentes o en desarrollo a adoptar políticas que les sean más favorables para ellos? Está claro que el planeta necesita alejarse del uso de los combustibles fósiles, para lo cual ya existen metas de reducción de emisiones, lo cual está contemplado en los diversos compromisos adquiridos en el marco del Acuerdo de Paris (2015), del cual Honduras también es signatario. Concretamente, se deben analizar las implicaciones económicas, incluyendo análisis de externalidades, costos sociales, y precios sombra; además de las implicaciones ambientales para cuantificar y proyectar los beneficios de cambiar de un tipo de sistema lineal a uno circular para así conocer sus costos de transacción y proyectar sus beneficios a nivel nacional.

\section{LITERATURA CITADA}

Alvarado, E. (2008). Segundo inventario nacional de bienes y servicios ambientales. SERNA, Tegucigalpa, Honduras. $80 \mathrm{p}$.

Acuña, B., Loaiza, S., Mendoza Corrales, R. y Chirinda, N. (2020). Sistema agroforestal Quesungual como fuente natural de emisiones de gases de efecto invernadero en la microcuenca Tecomapa, Somotillo-Nicaragua. La Calera, 20(35), 95-102. https://lacalera.una.edu.ni https://doi.org/10.5377/calera.v20i35.10217

Banco Central de Honduras (2020). Honduras en cifras 2018-2020. Disponible en: https://www.bch.hn/estadisticos/ GIE/LIBHonduras\%20en\%20cifras/Honduras\%20en\%20Cifras\%202018-2020.pdf

Blanco-Orozco, N., Zúniga-González, C., Torres, J., Arce-Díaz, E., Martínez-Andrade, E., Dios-Palomares, R., Quiros-Madrigal, O., \& Rueda-Hernández, M. (2016). Análisis de seguridad y productividad del suministro de energía eléctrica en el sistema eléctrico de Nicaragua en el periodo comprendido desde el año 2010 hasta el 2018. Revista Iberoamericana De Bioeconomía Y Cambio Climático E-ISSN 2410-7980, 1(2), 20-52. https:// doi.org/10.5377/ribcc.v1i2.2476

Blanco-Orozco N. V; Zúniga-González, C. A.; Arce, E.; Jaramillo-Villanueva, J. L., Losilla-Solano, L.; Colón-García, A. P. (2014). Evaluación integral financiera, económica, social, ambiental y productividad del uso de bagazo 
de caña en la generación de energía Eléctrica en Nicaragua en ingenios no conectados a la red de energía eléctrica. Universitas (León). Revista Científica de la UNAN-León. 5 (20) $42-51$ https://doi.org/10.5377/ universitas.v5i2.2033

Bolaños Sigcho, E. (2020). Impacto de las prácticas agroecológicas sobre la conservación, incremento o interacción de servicios ecosistémicos en suelos agrícolas: Revisión de Literatura. Zamorano, Honduras. https:// bdigital.zamorano.edu/bitstream/11036/6743/1/IAD-2020-T004.pdf.

Borrego Camacho, D. (2020). Perspectivas socioambientales y experiencias en agroecología en familias campesinas lenca del norte de Intibucá, Honduras. [tesis]. Centro Agronomico Tropical de Investigacion y Enseñanza.

Catari, G., Martinez, L. (2020). Agenda de política pública y privada para la bioeconomía. Caso Honduras. IICA. Tegucigalpa, Honduras, 09/09/2020. https://www.iica.int/es/prensa/eventos/el-potencial-de-la-bioeconomiapara-la-agricultura-y-el-desarrollo-rural-de-honduras\#!\#presentations

Chavarría, H., Trigo, E. y Martínez, J. F. (2021). Políticas y Negocios para la Bioeconomía en ALC: Un proceso en marcha. C3-BIOECONOMY: Circular and Sustainable Bioeconomy. Publicación en línea avanzada. https:// doi.org/10.21071/c3b.vi1.13150

Chen Zhang Robert Wohlhueter Han Zhang. (2016). Genetically modified foods: A critical review of their promise and problems. Food Science and Human Wellness. Volume 5, Issue 3, September 2016, Pages 116-123. https:// doi.org/10.1016/j.fshw.2016.04.002

Cherrett, L.A., y Welches E. (2019). El sistema Quesungual en Honduras Una alternativa a la tala y quema. En: Leisa. Revista de agroecologia 18 (3). https://www.leisa-al.org/web/index.php/volumen-18-numero-3/2240-elsistema-quesungual-en-honduras-una-alternativa-a-la-tala-y-quema

Dominguez, R., Sanchez, J., Sunkel, O., Samaniego, J. L. y Leon, M. (2019). Recursos naturales, medio ambiente y sostenibilidad: 70 años de pensamiento de la CEPAL. Libros de la CEPAL. Comisión Económica para América Latina y el Caribe. https://doi.org/10.18356/b89f0453-es

FAO. (2018). La agricultura mundial en la perspectiva del año 2050. Roma.

Fortin, M. T. (2021). Casos exitosos de aprovechamiento de la bioeconomía en la agricultura: Diversificación e innovación de productos de café en la finca Los Catadores. http://repositorio.iica.int/bitstream/ handle/11324/17529/BVE21078009e.pdf?sequence=1\&isAllowed $=y$

Gonzalez, C. A. Z. (2011). Comparisons of LSMS-ISA data collection and dissemination efforts in Central America. Journal of development and Agricultural Economics, 3(8), 353-361.

Hands, M. 2021. The search for a sustainable alternative to slash-and-burn agriculture in the World's rain forests: the Guama Model and its implementation. R. Soc. Open Sci. 8:201204. https://doi.org/10.1098/rsos.201204

Instituto Interamericano de Cooperación para la Agricultura (IICA). (2020). Tecnologías de bioeconomía para valorizar residuos y desperdicios: oportunidades de negocio para la agricultura familiar. IICA. San Jose, Costa Rica.

López-González, Á. S., Zúniga-González, C. A., López, M. R., Quirós-Madrigal, O. J., Colón-García, A. P., NavasCalderón, J., ... \& Rangel-Cura, R. A. (2015). State of the art for measuring productivity and technical efficiency in Latin America: Nicaragua Case. Revista Iberoamericana de Bieoconomía y Cambio Climático, 1(1131-2016-92270), 77-98. https://doi.org/10.5377/ribcc.v1i2.2478

Milán Pérez, J., \& Zúniga-Gonzalez, C. (2021). Necesidades de investigación y transferencia de tecnologías sobre cambio climático en Nicaragua: Una oportunidad en la Bioeconomía. Revista Iberoamericana De Bioeconomía Y Cambio Climático E-ISSN 2410-7980, 7(13), 1518-1543. https://doi.org/10.5377/ribcc.v7i13.11270

Marinero-Orantes, E., Vargas Cañas, J., Catari, G., Martínez, L., Sardiñas Gómez, O., \& Zúniga González, C. (2015). Análisis de la agenda pública y privada de la Bioeconomía en Centroamérica y el Caribe: Estudios de Caso de El Salvador, Honduras, Cuba y Nicaragua. Revista Iberoamericana De Bioeconomía Y Cambio Climático E-ISSN 2410-7980, 1(1), 242-284. https://doi.org/10.5377/ribcc.v1i1.2151

Macias Aragones, M., Giron Dominguez, C., Nieto Fajardo, M., Chavrier, N., Paez Rodriguez, D., Ureña Mayenco, M., Moreno Garcia, J. C., Garcia Alegre, M. y Viña Nieto, G. (2020). Tecnologías de bioeconomía para valorizar residuos y desperdicios: oportunidades de negocio para la agricultura familiar. 
Marques Almeida, E., Prat, J., Vargas-Moreno, J. C. y Acevedo, M. C. (2019). Honduras: Un enfoque territorial para el desarrollo. Inter-American Development Bank. https://doi.org/10.18235/0001679

Medina, L. (2018). Estrategia nacional de uso y manejo de fuego 20182028 y su plan de acción. Tegucigalpa: CLIFOR

Ordoñez, L. J. y Ferrufino Acosta, L. (2020). NOTA CIENTÍFICA: ESPECIES VEGETALES USADAS PARA LA BISUTERÍA EN HONDURAS. Etnobiologia, 18(1), 59-64. https://revistaetnobiologia.mx/index.php/etno/ article/view/356/341

Rangel Cura, R., Zúniga González, C., Colón García, A., Losilla Solano, L., \& Berrios-Zepeda, R. (2015). Medición de la contribución de la bioeconomía en América Latina: caso Cuba. Revista Iberoamericana De Bioeconomía Y Cambio Climático E-ISSN 2410-7980, 1(1), 223-240. https://doi.org/10.5377/ribcc.v1i1.2150

Ridher Ronaldo, Nina López; Dennis Gustavo, Toc Mó, (2020). Determinación de la resistencia de Parthenium hysterophorus L. al glifosato en Honduras. Tesis. Zamorano, Honduras. https://bdigital.zamorano.edu/ bitstream/11036/6826/1/CPA-2020-T105.pdf

Rodríguez, A. G., Mondaini, A. O. y Hitschfeld, M. A. (2017). Bioeconomía en América Latina y el Caribe: Contexto global y regional y perspectivas.

Rodríguez, A. (2020). La Bioeconomía: Oportunidades y desafíos para el desarrollo rural y agroindustrial en América Latina y el Caribe. En: Boletín CEPAL, FAO, IICA, Última comprobación el 12/07/2020.

Rodríguez, A; Rodrigues, M; Sotomayor, O. (2019). "Hacia una bioeconomía sostenible en América Latina y el Caribe: elementos para una visión regional", serie Recursos Naturales y Desarrollo, No 191 (LC/TS.2019/25)

Ruiz Muller, M; Trinidad, C.; Vargas, S. 2020. Políticas Públicas y Cambio Climático en América Latina. Recuento de la Arquitectura Institucional y Legal para la Implementación Efectiva. En: Boletín Política comercial y ambiental (10). Disponible en línea en https://www.kas.de/documents/273477/5442457/Politicas+publicas+y+Cambio+Climatico+en +ALC_Vf.pdf/95e5118f-0de9-5e31-884a-e8b565da221a?version=1.0\&t $=1592926315552$

Sánchez, Á. S., Melchor, G. I. H., Cruz, J. M. Z., González, C. A. Z., \& Galarza, J. L. S. (2018). Mangrove restoration an economical alternative for generating incomes. In Towards a Sustainable Bioeconomy: Principles, Challenges and Perspectives (pp. 307-317). Springer, Cham. https://doi.org/10.1007/978-3-319-73028-8_16

Silva, A. (2019). Experiencia en conservación de recursos naturales y pago por servicios ambientales en la micro cuenca Santa Isabel, Namasigüe, Choluteca. edit. por Marco Tulio Fortin. IICA. Honduras.

Sierra Figueredo, P., Pérez Piñeiro, A., Durán Zarabozo, O., Castellanos-Pontenciano, B., \& Zaldivar Cruz, J. (2015). Aspectos a valorar dentro de la bioeconomía y su sendero de eco - intensificación para el buen desempeño de la actividad apícola ante la variabilidad de la Actividad Solar y Geomagnética y los cambios climáticos. Revista Iberoamericana De Bioeconomía Y Cambio Climático E-ISSN 2410-7980, 1(1), 207-222. https:// doi.org/10.5377/ribcc.v1i1.2149

Secretaría de Energía, Recursos Naturales, Ambiente y Minas (MiAmbiente+). 2015. Inventario Nacional de Gases de Efecto Invernadero de Honduras INGEI-Serie 2005-2015.

Trigo, E; Henry, G. (2013=. Bioeconomy Working Paper No. 2013-01. Towards bioeconomy development in Latin America and the Caribbean. alcue-kbbe Project

Vargas-Hernández, José G., Pallagst, K., Hammer, P. (2018) Bioeconomía en la encrucijada del desarrollo sostenible. En: Rev. Iberoam. Bioecon. Cambio Clim. 4 (7), pág. 800-815. DOI: https://doi.org/10.5377/ribcc.v4i7.5952

Zuniga González, C. A. (2020). Crecimiento de la productividad total de los factores en la agricultura: análisis del índice de Malmquist de 14 países, 1979-2008. REICE: Revista Electrónica De Investigación En Ciencias Económicas, 8(16), 68-97. https://doi.org/10.5377/reice.v8i16.10661

Zúniga-González, C. A., Durán Zarabozo, O., Dios Palomares, R., Sol Sánchez, A., Guzman Moreno, M. A., Quiros, O., \& Montoya Gaviria, G. D. J. (2014). Estado del arte de la bioeconomía y el cambio climático (No. 1133-2016-92457, pp. 20-329). DOI 10.22004/ag.econ.168356 ISBN : 978-99924-28-40-5

Zúniga-González, C. A.; Sardiñas Gómez, O.; Quiros, O.;Sol Sánchez, A.;Blanco Roa, N. E.;Salazar Antón, W.;ColónGarcía, A. P.;Rangel Cura, R. A. (2014a): Bioeconomy Productive Path: A review in LAC. Nicaragua. Book/ Chapter DOI10.22004/ag.econ.168358 ISBN : 978-99924-28-40-5 
Secretaría de Energía, Recursos Naturales, Ambiente y Minas (MiAmbiente+). 2017. Estrategia Nacional de biodiversidad de Honduras. Secretaría de Energía, Recursos Naturales, Ambiente y Minas (MiAmbiente+). Tegucigalpa, Honduras, https://www.cbd.int/doc/world/hn/hn-nbsap-v2-es.pdf

Agro Bio (2016). Adopcio\#n de cultivos transge\#nicos alcanzan en 2016 su pico ma\#s alto en el mundo (2016). https://www.agrobio.org/adopcion-cultivos-transgenicos-alcanzan-2016-pico-mas-alto-mundo/

Instituto Nacional de Estadistica (2020) https://www.ine.gob.hn/V3/

Banco Interamericano de Desarrollo (2019. Informe anual 2019. https://publications.iadb.org/es/bancointeramericano-de-desarrollo-informe-anual-2019-estados-financieros

Moreno Banegas, G. (2012). Caracterización de la respuesta a la temperatura en la germinación de semillas de Jatropha curcas [en línea]. Trabajo Final de Ingeniería en Producción Agropecuaria. Facultad de Ciencias Agrarias. Universidad Católica Argentina. Disponible en: http://bibliotecadigital.uca.edu.ar/repositorio/tesis/ caracterizacion-respuesta-temperatura-germinacion.pdf [27-10-2021]

Aguilar Rivera, N. (2014). Reconversión de la cadena agroindustrial de la caña de azúcar en Veracruz México. Nova scientia, 6(12), 125-161.

Programa Especial para la Seguridad Alimentaria (PESA) Centroamérica (2005). Sistemas de riego. https:// www.fao.org/in-action/pesa-centroamerica/es/

Gómez-Calderón, N; Villagra-Mendoza, K; Solorzano-Quintana, M. La labranza mecanizada y su impacto en la conservación del suelo (revisión literaria). Tecnología en Marcha. Vol. 31-1. Enero-Marzo 2018. Pág 170-180

\section{Anexo}

\section{Tabla 1}

Senderos productivos de la bioeconomía 
Revista Iberoamericana de Bioeconomía y Cambio Climático, 2021, 7(14), Agosto-Diciembre, ISSN: 241...

TABLA 1

Senderos productivos de la bioeconomía

\begin{tabular}{|c|c|}
\hline Sendero & Características \\
\hline $\begin{array}{l}\text { Valorización de la diversidad } \\
\text { biológica }\end{array}$ & $\begin{array}{l}\text { Dar valor a los recursos de la } \\
\text { biodiversidad a través de procesar o } \\
\text { transformar de manera innovadora, } \\
\text { desarrollar mercados, utilizar rasgos } \\
\text { funcionales y desarrollar productos } \\
\text { locales novedosos, entre otros } \\
\text { aspectos. }\end{array}$ \\
\hline Eco intensificación & $\begin{array}{l}\text { Reducir el impacto ambiental a } \\
\text { través de prácticas innovadoras en } \\
\text { los sistemas de producción sin } \\
\text { disminuir su eficiencia (labranza } \\
\text { cero, bioinsumos, entre otras). }\end{array}$ \\
\hline $\begin{array}{l}\text { Aplicaciones de } \\
\text { biotecnología (productos y } \\
\text { procesos) }\end{array}$ & $\begin{array}{l}\text { Cultivo de tejidos, selección asistida } \\
\text { por marcadores semillas } \\
\text { genéticamente modificadas, } \\
\text { diagnóstico molecular, } \\
\text { mejoramiento de reproducción } \\
\text { animal utilizando técnicas } \\
\text { moleculares, microorganismos, } \\
\text { enzimas transformadas y levaduras, } \\
\text { etc. }\end{array}$ \\
\hline Servicios eco sistémicos & $\begin{array}{l}\text { Provienen de las funciones de los } \\
\text { ecosistemas como un conjunto. Son } \\
\text { servicios que no tienen valor en los } \\
\text { mercados tradicionales y por lo } \\
\text { tanto se debe recurrir a técnicas } \\
\text { económicas para asignarles un valor } \\
\text { de conservación. Destacan el } \\
\text { servicio hídrico, secuestro de } \\
\text { carbono, conservación de } \\
\text { biodiversidad, y belleza escénica } \\
\text { como insumo para el ecoturismo. }\end{array}$ \\
\hline $\begin{array}{l}\text { Eficacia de las cadenas de } \\
\text { valor }\end{array}$ & $\begin{array}{l}\text { Disminuir pérdidas por el } \\
\text { desaprovechamiento de residuos en } \\
\text { cada uno de sus eslabones, para } \\
\text { enfocarse en la transformación de } \\
\text { bioproductos que mejoren la } \\
\text { competitividad de la cadena. }\end{array}$ \\
\hline Biorrefinerías y bioproductos & $\begin{array}{l}\text { Sector de la bioenergía y de los } \\
\text { procesos cuyo objetivo es sustituir el } \\
\text { combustible fósil. Para tal caso, las } \\
\text { plantas de bioetanol, biodiesel, } \\
\text { biogás, y las distintas actividades } \\
\text { vinculadas con la química verde. }\end{array}$ \\
\hline
\end{tabular}

Fuente: Elaboración propia 